\title{
REFLECTION FROM A GOLD SPUTTERED THIN LAYER
}

\author{
Pavel Buchar ${ }^{*}$ Jan Macháćc , Ján Zehentner*, Petr Slepička **, Václav Švorčík
}

\begin{abstract}
This paper presents the results of an investigation of a thin layer of gold sputtered on a dielectric foil. This layer is modeled by nanometer-scale spherical particles that are separated at short deposition times and later touch each other, forming a continuous layer. Four ways of analyzing the structure are introduced. The first method describes the spheres by their electric dipole moment, while the second, newly proposed method, takes into account the capacity between adjacent spheres, and the third method assumes the layer as homogeneous and considers its reduced conductivity due to the behavior of electrons in thin films. The CST Microwave Studio model, which takes into account the random distribution of particles, offers the reflection coefficient that best fits measured data.
\end{abstract}

\section{INTRODUCTION}

Thin metallic layers have been gaining significant importance, e.g., in the field of so-called active packaging [1] and electromagnetic shielding [2]. The behavior of a layer of metallic nanometer-scale spheres [3] and rings [4] has been investigated at optical frequencies. The response of thin metallic layers at microwave frequencies has been investigated by several authors. It was shown in [5] that even a very thin layer may absorb up to $50 \%$ of the incident electromagnetic wave energy. The influence of layer thickness on effective conductivity was studied in [6].

A thin sputtered gold layer was experimentally investigated at microwave frequencies in [7]. It was found that there is a small reflection from a layer of disconnected gold nano-particles for short sputtering times. For long sputtering times when the gold layer up to tens of nanometers in thickness is homogeneous, the reflection is high. The transition between these two states of reflection was recorded.

The thin metallic layer is represented in this paper as a 2D array of spherical gold particles. The process of gradual depositing of metal by sputtering is simulated by decreasing the distance between particles, which is equivalent to increasing their number. Their interaction with an electromagnetic wave is interpreted in four ways. The first model taken from [8] analyses the electromagnetic field interaction with the electric dipole moment of the particles. The second, newly proposed model, is based on the capacities between spheres. Next we consider the layer to be homogeneous, and take into account the effect of decreasing conductivity in very thin films [6], which gives results valid for longer sputtering times. Finally, the layer of gold particles is modeled using the CST Microwave Studio (MWS). Unlike the above-mentioned three models, the MWS model accounts for a random distribution of particles over the analyzed aperture, which gives the most relevant results. All resulting curves of the reflection coefficient with respect to sputtering time are compared with the measured data. The aim of this work is to approximate the measured reflection coefficient [7] for the whole range of investigated sputtering times.

\section{MODELS OF THE SPUTTERED LAYER}

The investigated gold layer was sputtered on a polymer foil. However, the presented models consider only the metal layer, which is represented by equivalent surface impedance $Z_{g}$. In the case of a purely resistive homogeneous layer, this impedance is reduced to its square resistivity.

\section{A. Array model}

This model is taken from [8]. It considers a regular rectangular 2D array of electrically small particles polarized by the electric field. Only dipoles will be excited by an external field, and multipoles are neglected. The arrangement is sketched in Fig. 1. The particles are placed with distance $a$ and are illuminated by a perpendicularly incident plane wave $\mathbf{E}_{\text {ext }}, \mathbf{H}_{\text {ext }}$ with the wave vector $\mathbf{k}_{\text {ext }}$. The particles are described by their polarizability $\alpha$ [8], which links the electrical dipole moment $\mathbf{p}$ to the local electric field $\mathbf{E}_{\mathrm{loc}}$, determined by the sum of the field of the

\footnotetext{
${ }^{*}$ Czech Technical University, Technicka 2, 16627 Prague 6, Czech Republic, phone: +420 224352279, e-mail: bucharp@ffel.cvut.cz, machac@,fel.cvut.cz, zehent@,fel.cvut.cz

${ }^{* *}$ Institute of Chemical Technology, Technicka 1, 16628 Prague 6, Czech Republic, phone: +420 220445149, e-mail: petr.slepicka@,vscht.cz, vaclav.svorcik@,vscht.cz
} 
incident wave and of the field produced by all other particles, $\mathbf{p}=\alpha \mathbf{E}_{l o c}$. According to [8], the equivalent impedance of the array is

$$
Z_{g}=-j \frac{a^{2}}{\omega}\left[\operatorname{Re}\left(\frac{1}{\alpha}-\beta\right)+j\left(\operatorname{Im}\left(\frac{1}{\alpha}\right)-\frac{k_{e x t}^{3}}{6 \pi \varepsilon_{0}}\right)\right],
$$

where $\omega$ is angular frequency and $\beta$ is the so-called interaction constant [8].

\section{B. Capacitive and metallic layer models, combined model}

A capacitive model anticipates only a capacitive character of the equivalent impedance due to the mutual capacity between spheres. The sphere conductivity is assumed infinite. Like the layer model, the capacitive model is also applicable at short sputtering times when the particles are still separated. According to [10], the mutual capacity $C$ between two spheres with radius $r$ and distance of their centers $a$, Fig. 1, is given by the series

$$
C=4 \pi \varepsilon_{0} r^{2}\left(\frac{1}{a}+\frac{r^{2}}{a^{3}-2 a r^{2}}+\frac{r^{4}}{a^{5}-4 a^{3} r^{2}+3 a r^{4}}+\ldots\right) .
$$

The equivalent impedance is then

$$
Z_{g}=1 /(j \omega C)
$$

A thin metallic layer model was taken from [6]. Unlike earlier models describing the behavior of individual metallic nano-particles, this model considers the investigated structure as a continuous layer, and is thus applicable at long sputtering times. It was shown in [6] that if the layer thickness $h$ is not greater than the electron mean free path, the conductivity is reduced to

$$
\sigma=\sigma_{0}\left(1-\frac{3}{8 x}+\frac{e^{-x}}{16 x}\left(6-10 x-x^{2}+x^{3}\right)+\frac{x}{16}\left(12-x^{2}\right) \int_{x}^{\infty} \frac{e^{-u}}{u} d u\right)
$$

where $\sigma$ is reduced conductivity, $\sigma_{0}$ is the conductivity of the bulk material, $x=h / \lambda$, where $\lambda$ is the electron mean free path. For gold $\sigma_{0}=4.110^{7} \mathrm{~S} / \mathrm{m}, \lambda=0.057 \mu \mathrm{m}$. The equivalent impedance is now the layer square resistivity $R_{S}$

$$
Z_{g}=R_{S}=1 /(\sigma h)
$$

The two last-mentioned models can be combined in order to describe both the capacitive character of separated metallic nanoparticles and the resistivity of a homogeneous metallic layer. The equivalent impedance $Z_{g}$ is now a series combination of (3) and (5).

\section{CST Microwave Studio model}

The layer was modeled using the CST Microwave Studio, which directly computes scattering parameters. The layer of spheres was analyzed in a parallel plate waveguide terminated from the sides by perfect magnetic walls. The spheres were either regularly or randomly distributed over the aperture of this waveguide. Random distribution of spheres is more convenient, as it excludes mutual touching of all particles at the same moment when their number is increased. Mutual touching causes a step increase in the reflection coefficient, as the structure is at this time short cut by the connected spheres.

\section{REFLECTION OF THE INVESTIGATED THIN LAYER}

The investigated sputtered gold layers were tested in a waveguide [7]. In the individual models we assumed only the sputtered gold layer itself without the polymer foil. We replaced this layer by equivalent impedance $Z_{g}$, shown in an equivalent circuit in Fig. 2. Alternatively, this impedance can be described by the scattering parameters. According to [9]

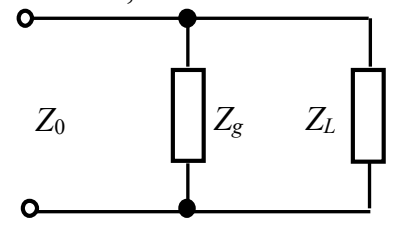

Fig. 2. The equivalent circuit of the metallic layer arrangement in a waveguide. 


$$
S_{11}=S_{22}=-1 /\left(1+\frac{2 Z_{g}}{Z_{0}}\right), \quad S_{21}=S_{12}=1 /\left(\frac{Z_{0}}{2 Z_{g}}+1\right),
$$

where $Z_{0}$ is the characteristic impedance of the waveguide. The influence of the polymer foil and the matched load terminating the waveguide [7], is considered by impedance $Z_{L}$, Fig. 2. $Z_{L}$ is related to the reflection coefficient $\Gamma$ by

$$
\Gamma=\frac{Z_{L}-Z_{0}}{Z_{L}+Z_{0}} .
$$

The value of $\mid \Gamma=0.018$ or $-34.9 \mathrm{~dB}$ is known from the measurement of the foil without the metallic layer, i.e., for zero sputtering time [7]. When the scattering parameters (6) are known, the resulting value of the reflection coefficient may subsequently be computed according to the formula [9]

$$
R=S_{11}+\frac{S_{21} S_{12} \Gamma}{1-S_{22} \Gamma} .
$$

This reflection coefficient $R$ is finally compared with the measured values of the reflection coefficient taken from [7].

\section{DISCUSSION OF RESULTS}

\section{A. Regular distribution of spheres}

At first a regular 2D array of gold spheres with constant radius was considered. The distance between the spheres was reduced successively in order to simulate gradual sputtering, during which the number of particles increases in time and their distance decreases. The equivalent thickness of the layer can be determined by dividing of the volume of the sphere by the area of one cell

$$
h=\frac{4}{3} \pi r^{3} / a^{2} .
$$

Sputtering time $t$ can subsequently be computed from $h$ according to [7]

$$
h(t)=0.16768 t .
$$

The sphere radius $r=5.75 \mathrm{~nm}$ was chosen to give a good approximation of the measured data. In Fig. 3, the time dependences of reflection coefficient $R(8)$ obtained by the three methods are shown. The measured data is taken from [7]. It can be observed that for short times the calculated reflectivity fits the measured data well. Differences occur in the fast increase region and beyond, when the layer is homogeneous. The curve obtained by measurement grows more slowly than the curves obtained by modeling. This is due to the fact that the models assume a regular distribution of spheres and the fast growth in the reflection coefficient corresponds to mutual touching of all spheres.

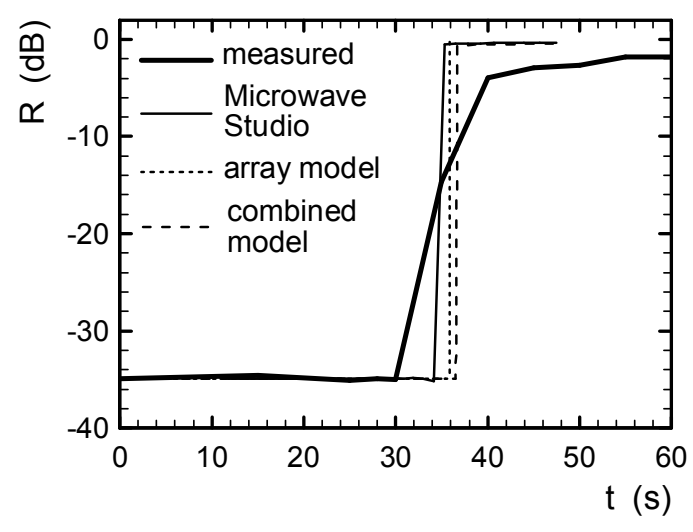

\section{B. Random distribution of spheres}

In order to remove the above-mentioned discrepancies, spheres were randomly distributed over the foil in the CST

Fig. 3. Sputtering time dependence of reflection coefficient $R$, regular sphere distribution.

Microwave Studio simulation model. The sphere radius is constant and the number of spheres $n$ changes in order to simulate the gradual sputtering process. With increasing number of spheres on the aperture $S$, their average distance $a=\sqrt{S / n}$ gradually decreases. This value is used in (9) and the corresponding sputtering time follows from (10). The spheres do not touch each other simultaneously, as in the case of regular distribution. This assures slow growth of the reflection coefficient in the area where the sputtered gold layer gradually becomes homogeneous. The resulting sputtering time dependence of the reflection coefficient obtained by averaging 30 simulations is shown in Fig. 4. This averaging makes the curve of the reflection coefficient smooth by reducing the influence of 
the non-uniform distribution of the finite number of particles over a finite area. The calculated reflection coefficient provides a better approximation of measured $R$ in its rapidly increasing region than those models that assume regular sphere distribution.

\section{CONCLUSION}

The paper presents models of a gold layer sputtered on a dielectric foil. The gold was replaced by a 2D array of conducting spheres either regularly or randomly distributed. The decreasing distance or increasing number of spheres simulates the gradual deposition of gold. The layer of regularly distributed spheres was modeled by the array model taken from [8], and by the newly proposed combined capacitive-layer model. The latter considers both the capacity between the

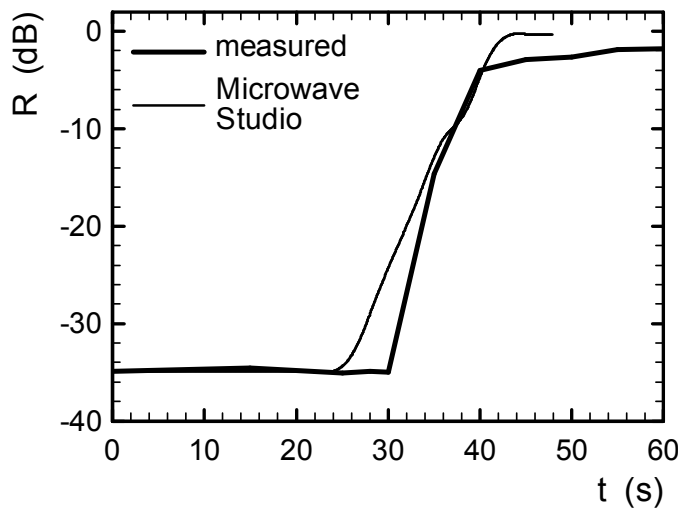

Fig. 4. Sputtering time dependence of the reflection coefficient $R$, random sphere distribution. individual spheres and the square resistivity of the layer. Simulation by the CST Microwave Studio was performed for both regular and random distribution of spheres.

All models provide a good approximation of the measured data for low sputtering times, when the structure is not homogeneous. In this case the reflection coefficient is approx. $-35 \mathrm{~dB}$. For sputtering times of 30-40 $\mathrm{s}$, when the structure gradually becomes homogeneous, there are some differences. The results obtained from regular distribution of spheres are very similar to each other. They show, however, a very rapid increase in the reflection coefficient due to the mutual contact of the spheres, whereas the measured reflection coefficient increases more slowly. This discrepancy was partly removed by considering random distribution of spheres in the CST Microwave Studio. The resulting curve increases more slowly and approximates the measured data better. For sputtering times above $40 \mathrm{~s}$, the array model is no longer valid. The combined model and the CST Microwave Studio model fit each other well, and the reflection coefficient is approx. between -0.5 and $-0.3 \mathrm{~dB}$, whereas the measurement shows the reflection coefficient from about -4 to $-1 \mathrm{~dB}$. This difference is probably due to unknown phenomena occurring in nanometer-scale objects, which are not considered by our macroscopic models.

In comparison with analytic models, the CST Microwave Studio has the disadvantage of not being analytic and requiring a long simulation time. However, the only CST Microwave Studio model is able to consider the random distribution of conducting spheres, and thus it provides reflectivity best fitting the measured reflection coefficient.

Our investigation is important for producing a sputtered gold layer with defined impedance, e.g., in the domain of active packaging and electromagnetic shielding.

\section{ACKNOWLEDGEMENT}

This work was supported by the Grant Agency of the Czech Republic under project 102/03/0449 "New circuit devices for communication technology".

\section{References}

[1] V. V. Yakovlev, "Improving Quality of Microwave Heating by Packaging - Analytical Approach," 2001 ASAE Annual International Meeting Sponsored by ASAE, Sacramento, CA, paper 01-6149, July-August 2001.

[2] S. Pellicori, "Transparent Electromagnetic Compatibility Coatings," Coating Material News, vol. 13, March 2003.

[3] G. Bosi, "Transmission of a thin film of spherical particles on a dielectric substrate: the concept of effective medium revisited," Journal of the Optical Society of America B, vol. 9, February 1992.

[4] J. Aizpurua et al., "Light Scattering in Gold nanorings," Journal of Quantitative Spectroscopy and Radiative Transfer, vol. 89, pp. 11-16, 2004

[5] H. Bosman, Y. Y. Lau, R. M. Gilgenbach, "Microwave absorption on a thin film," Applied Physics Letters, vol. 82, pp. 13531355, March 2003.

[6] R. C. Hansen, W. T. Pawlewicz, "Effective Conductivity and Microwave Reflectivity of Thin Metallic Films," IEEE Transaction on Microwave Theory and Techniques, vol. 82, pp. 2064-2066, November 1982.

[7] J. Zehentner et al., "Effective Conductivity and Microwave Reflectivity of Thin Metallic Films," EMFM 2004 Proceedings, vol. 1, pp. 105-108, May 2004

[8] S. Tretyakov, Analytical Modeling in Applied Electromagnetics, Norwood: ARTEC HOUSE INC., 2000.

[9] F. Nibler, High-frequency circuit engineering, London: Institution of Electrical Engineers, 1996.

[10] F. M. Erickson, "The Capacitance Between Two Spheres," http://www.ttc-cmc.net/ fme/spheres.11-03-99.ps.gz, 1999 